\title{
On Bayesian Measures of Evidential Support: Theoretical and Empirical Issues*
}

\author{
Vincenzo Crupi, Katya Tentori, and Michel Gonzalezł:
}

Epistemologists and philosophers of science have often attempted to express formally the impact of a piece of evidence on the credibility of a hypothesis. In this paper we will focus on the Bayesian approach to evidential support. We will propose a new formal treatment of the notion of degree of confirmation and we will argue that it overcomes some limitations of the currently available approaches on two grounds: (i) a theoretical analysis of the confirmation relation seen as an extension of logical deduction and (ii) an empirical comparison of competing measures in an experimental inquiry concerning inductive reasoning in a probabilistic setting.

1. Rival Bayesian Measures of Confirmation. Judgments concerning the support that a piece of information brings to a hypothesis are commonly required in scientific research as well as in other domains (medicine, law), and a major aim of a theory of inductive reasoning is to provide a proper foundation to such judgments.

Within the Bayesian approach to inductive reasoning, an attempt to measure degrees of confirmation, or evidential support, should reflect, and extend, a basic qualitative view of confirmation-labeled the "clas-

*Received March 2006; revised September 2006.

$\dagger$ To contact the authors, please write to: Vincenzo Crupi, Department of Arts and Design, University IUAV of Venice, Convento delle Terese, Dorsoduro 2206, Venice 30123, ITALY; e-mail: vincenzo.crupi@iuav.it; Katya Tentori, Department of Cognition Science and Education, University of Trento, via Matteo del Ben 5, Rovereto, Trento 38068, ITALY; e-mail: katya.tentori@unitn.it; Michel Gonzalez, Laboratoire de Psychologie Cognitive, University of Provence \& CNRS, Place Victor Hugo, Marseille 13331, FRANCE; e-mail: michel.gonzalez@up.univ-mrs.fr.

\$We thank Roberto Festa, Branden Fitelson, Theo Kuipers, Daniel Osherson, and two anonymous referees for comments on previous versions of this paper. Research was supported by PRIN 2005 grant Le dinamiche della conoscenza nella società dell'informazione and by a grant from the SMC/Fondazione Cassa di Risparmio di Trento e Rovereto.

Philosophy of Science, 74 (April 2007) pp. 229-252. 0031-8248/2007/7402-0003\$10.00

Copyright 2007 by the Philosophy of Science Association. All rights reserved. 
table 1. Alternative Bayesian Measures of Confirmation.

\begin{tabular}{ll}
\hline$D(e, h)=p(h \mid e)-p(h)$ & Carnap ([1950] 1962) \\
$S(e, h)=p(h \mid e)-p(h \mid \neg e)$ & Christensen (1999) \\
$M(e, h)=p(e \mid h)-p(e)$ & Mortimer (1988) \\
$N(e, h)=p(e \mid h)-p(e \mid \neg h)$ & Nozick (1981) \\
$C(e, h)=p(e \& h)-p(e) \cdot p(h)$ & Carnap ([1950] 1962) \\
$R(e, h)=[p(h \mid e) / p(h)]-1$ & Finch (1960) \\
$G(e, h)=1-[p(\neg h \mid e) / p(\neg h)]$ & Rips (2001) \\
$L(e, h)=[p(e \mid h)-p(e \mid \neg h)] /[p(e \mid h)+p(e \mid \neg h)]$ & Kemeny and Oppenheim (1952) \\
\hline
\end{tabular}

sificatory concept of confirmation" by Carnap ([1950] 1962, 21-22). This view identifies confirmation with an increase in the probability of the hypothesis (conclusion) $h$ provided by the piece of information (premise) $e$, neutrality with a lack of impact of $e$ on the probability of $h$, and disconfirmation with a decrease of such a probability as an effect of $e$. A common way to convey this distinction is to formalize the confirmation relation by a mathematical function $c(e, h)$, depending on probability values concerning $e$ and $h$, such that the following condition (BC) is satisfied (BC stands for "Bayesian confirmation"): ${ }^{1}$

$$
c(e, h)\left\{\begin{array}{lll}
>0 & \text { if } p(h \mid e)>p(h) & \text { [confirmation] } \\
=0 & \text { if } p(h \mid e)=p(h) & \text { [neutrality] } \\
<0 & \text { if } p(h \mid e)<p(h) & \text { [disconfirmation]. }
\end{array}\right.
$$

Condition (BC) does not put any constraint on the values to be assigned to confirmatory arguments (as long as such values are positive) or to disconfirmatory arguments (as long as such values are negative). Indeed, (BC) does not constrain the choice of one single measure of confirmation as the most adequate (a point emphasized by Fitelson [1999]). As a matter of fact, several nonequivalent measures can be, and have been, devised that map relevant probability values onto a number that is positive iff $p(h \mid e)>p(h)$, amounts to zero iff $p(h \mid e)=p(h)$, and is negative otherwise. Table 1 provides a catalogue collected from the literature.

Quantitative measures of confirmation such as those in Table 1 have the important property of allowing ordinal judgments concerning inductive strength, such as "hypothesis $h$ receives more empirical support by

1. A few remarks are in order here. First, confirmation should be properly conceived (at least) as a three-place relation involving, beyond $h$ and $e$, the relevant "background knowledge." However, this point will not affect the present discussion. Thus, for the sake of simplicity and without loss of generality, we will omit background knowledge from our notation. Second, throughout the paper we will assume that $e$ and $h$ are contingent (i.e., neither logically true nor logically false) and that the probability function $p$ is regular (i.e., that $p(x) \neq 0$ unless $x$ is logically false and $p(x) \neq 1$ unless $x$ is logically true). See Festa (1999) and Kuipers (2000) for discussions on how to handle with Bayesian means some limiting cases that have been excluded here. 
$e_{1}$ than by $e_{2}$ " or " $e$ confirms $h_{1}$ to a greater extent than $h_{2}$." Two measures of confirmation are ordinally equivalent iff they give the same answer to the following question: Which one, if any, has a higher confirmation value in any given pair of arguments? ${ }^{2}$ In this respect, Table 1 provides a representative sample, for most of the Bayesian confirmation measures that we have been able to identify in the literature, but have not included in our list, are ordinally equivalent to some measure that does appear in the list. $^{3}$

2. A First Adequacy Requirement. One way to handle the plurality of Bayesian measures of confirmation is to resort to the long-standing and traditional view of inductive logic as an "extension" of classical deductive logic (Carnap [1950] 1962). Consider a function $v$ construed on the basis of classical deductive logic and such that, for any argument $(e, h), v$ assigns it the same positive value (e.g., +1 ) iff $e \vDash h$ (i.e., iff $e$ implies $h$ ), an equivalent value with opposite sign $(-1)$ iff $e \vDash \neg h$ (i.e., iff $e$ refutes $h$ ), and value 0 otherwise. The relationships between the logical implication or refutation of $h$ by $e$ and the conditional probability of $h$ given $e$ yield that any Bayesian confirmation measure $c$ agrees with $v$ in the minimal

2. Formally, measure $c$ is ordinally equivalent to measure $c^{*}$ iff, for any pair of arguments $\left(e_{1}, h_{1}\right)$ and $\left(e_{2}, h_{2}\right), c\left(e_{1}, h_{1}\right) \gtreqless c\left(e_{2}, h_{2}\right)$ iff $c^{*}\left(e_{1}, h_{1}\right) \gtreqless c^{*}\left(e_{2}, h_{2}\right)$.

3. In particular, measures $R, G$, and $L$ in our list are ordinally equivalent, respectively, to the following well-known measures of confirmation:

$$
\begin{array}{ll}
R^{*}(e, h)=p(h \mid e) / p(h) & {[\text { Keynes 1921] }} \\
G^{*}(e, h)=p(\neg h) / p(\neg h \mid e) & {[\text { Gaifman 1979] }} \\
L^{*}(e, h)=p(e \mid h) / p(e \mid \neg h) & {[\text { Good 1950]. }}
\end{array}
$$

It may be shown that $R(e, h)=R^{*}(e, h)-1, G(e, h)=1-\left[1 / G^{*}(e, h)\right]$, and $L=$ $\left(L^{*}-1\right) /\left(L^{*}+1\right)$. Notice that (unlike $R, G$, and $\left.L\right) R^{*}, G^{*}$, and $L^{*}$ are always positive and identify 1 as the "neutrality" value, thus departing from condition (BC) above. A common strategy to have such measures satisfying condition (BC) is to apply logarithms (with base $>1$ ) to them. By this strategy, one again obtains measures ordinally equivalent, respectively, to our $R, G$, and $L$. However, by the use of logarithms, such measures are not defined when $p(h \mid e)=1$ and/or when $p(h \mid e)=0$.

Under rather weak assumptions, it can also be proved that Table 1 includes no pair of ordinally equivalent measures. In fact, there exist quite simple probability models such that the measures included in Table 1 yield eight mutually incompatible rankings of inductive strength. For instance, let it be the case that $p\left(e_{1}\right)=.03, p\left(e_{2}\right)=.01$, $p\left(e_{3}\right)=.65, p\left(e_{4}\right)=.52, p\left(h_{1}\right)=.02, p\left(h_{2}\right)=.15, p\left(h_{3}\right)=.50, p\left(h_{4}\right)=.70, p\left(h_{1} \mid e_{1}\right)=$ $.32, p\left(h_{2} \mid e_{2}\right)=.48, p\left(h_{3} \mid e_{3}\right)=.75$, and $p\left(h_{4} \mid e_{4}\right)=.98$. Then the following rankings obtain: $D\left(e_{2}, h_{2}\right)>D\left(e_{1}, h_{1}\right)>D\left(e_{4}, h_{4}\right)>D\left(e_{3}, h_{3}\right) ; S\left(e_{3}, h_{3}\right)>S\left(e_{4}, h_{4}\right)>S\left(e_{2}, h_{2}\right)>$ $S\left(e_{1}, h_{1}\right) ; M\left(e_{1}, h_{1}\right)>M\left(e_{3}, h_{3}\right)>M\left(e_{4}, h_{4}\right)>M\left(e_{2}, h_{2}\right) ; N\left(e_{4}, h_{4}\right)>N\left(e_{3}, h_{3}\right)>N\left(e_{1}, h_{1}\right)>$ $N\left(e_{2}, h_{2}\right) ; C\left(e_{3}, h_{3}\right)>C\left(e_{4}, h_{4}\right)>C\left(e_{1}, h_{1}\right)>C\left(e_{2}, h_{2}\right) ; R\left(e_{1}, h_{1}\right)>R\left(e_{2}, h_{2}\right)>R\left(e_{3}, h_{3}\right)>$ $R\left(e_{4}, h_{4}\right) ; G\left(e_{4}, h_{4}\right)>G\left(e_{3}, h_{3}\right)>G\left(e_{2}, h_{2}\right)>G\left(e_{1}, h_{1}\right) ; L\left(e_{1}, h_{1}\right)>L\left(e_{4}, h_{4}\right)>L\left(e_{2}, h_{2}\right)>$ $L\left(e_{3}, h_{3}\right)$ (computational details are omitted). 
TABLE 2.

\begin{tabular}{lcc}
\hline Measure & If $e \vDash h$ & If $e \vDash \neg h$ \\
\hline$D(e, h)$ & $p(\neg h)$ & $-p(h)$ \\
$S(e, h)$ & $p(\neg h) / p(\neg e)$ & $-p(h) / p(\neg e)$ \\
$M(e, h)$ & $p(e) \cdot[p(\neg h) / p(h)]$ & $-p(e)$ \\
$N(e, h)$ & $p(e) / p(h)$ & $-p(e) / p(\neg h)$ \\
$C(e, h)$ & $p(e) \cdot p(\neg h)$ & $-p(e) \cdot p(h)$ \\
$R(e, h)$ & $p(\neg h) / p(h)$ & -1 \\
$G(e, h)$ & 1 & $-p(h) / p(\neg h)$ \\
\hline
\end{tabular}

sense that if $v(e, h)$ is positive, the same is true of $c(e, h)$; and if $v(e, h)$ is negative, the same is true of $c(e, h)$. Then, consistency with the following principle may be posited as a plausible adequacy requirement for $c$ (here, "Ex" stands for the "extension" from the deductive to the inductive domain):

$\left(\mathrm{Ex}_{1}\right)$ If $v\left(e_{1}, h_{1}\right)>v\left(e_{2}, h_{2}\right)$, then $c\left(e_{1}, h_{1}\right)>c\left(e_{2}, h_{2}\right)$.

Fulfillment of principle $\left(\mathrm{Ex}_{1}\right)$ guarantees that, by $c$, any conclusively confirmatory argument $(e, h)$ (i.e., such that $e \vDash h$ ) is assigned a higher value than any argument that is not conclusively confirmatory and any conclusively disconfirmatory argument $(e, h)$ (i.e., such that $e \vDash \neg h$ ) is assigned a lower value than any argument that is not conclusively disconfirmatory.

Remarkably, it can be proved (see below) that only one among the measures of confirmation listed in Table 1 actually fulfills $\left(\mathrm{Ex}_{1}\right)$, that is, $L .^{4}$ It turns out, however, that there is a rather simple way to obtain a measure of confirmation that does fulfill $\left(\mathrm{Ex}_{1}\right)$ from either $D, S, M, N$, $C, R$, or $G$. To see this, let's consider the formulas to which such measures can be reduced when $e \vDash h$ and when $e \vDash \neg h$, respectively, as reported in Table 2.

Now, for each measure in Table 2, let's employ the expressions appearing in the second and third columns (taken in absolute value) to

4. Fitelson (2006) - an advocate of confirmation measures ordinally equivalent to $L-$ has labeled "logicality" the following desideratum: $c(e, h)$ is maximal (minimal) when $e \vDash h(e \vDash \neg h)$. (Also see Kemeny and Oppenheim [1952] for the first proposal of measure $L$ as derived from a set of adequacy requirements including an early statement of logicality.) Notably, logicality and $\left(\mathrm{Ex}_{1}\right)$ are independent. On the one hand, logicality, but not $\left(\mathrm{Ex}_{1}\right)$, is violated by measures assigning different confirmation values to any two deductive arguments (for both to be "maximal," they actually have to be equal). On the other hand, a measure assigning value, say, +1 in any case of confirmation, -1 in any case of disconfirmation, and 0 in case of neutrality would violate $\left(\operatorname{Ex}_{1}\right)$ but not logicality. (We are indebted to an anonymous referee for the latter remark.) 
"normalize" degrees of positive and negative evidential support, respectively, as follows:

$$
\begin{aligned}
D_{\text {norm }}(e, h) & = \begin{cases}D(e, h) / p(\neg h) & \text { if } p(h \mid e) \geq p(h) \\
D(e, h) / p(h) & \text { otherwise, }\end{cases} \\
S_{\text {norm }}(e, h) & = \begin{cases}S(e, h) /[p(\neg h) / p(\neg e)] & \text { if } p(h \mid e) \geq p(h) \\
S(e, h) /[p(h) / p(\neg e)] & \text { otherwise, }\end{cases} \\
M_{\text {norm }}(e, h) & = \begin{cases}M(e, h) /\{p(e) \cdot[p(\neg h) / p(h)]\} & \text { if } p(h \mid e) \geq p(h) \\
M(e, h) / p(e) & \text { otherwise, }\end{cases} \\
N_{\text {norm }}(e, h) & = \begin{cases}N(e, h) /[p(e) / p(h)] & \text { if } p(h \mid e) \geq p(h) \\
N(e, h) /[p(e) / p(\neg h)] & \text { otherwise, }\end{cases} \\
C_{\text {norm }}(e, h) & = \begin{cases}C(e, h) /[p(e) \cdot p(\neg h)] & \text { if } p(h \mid e) \geq p(h) \\
C(e, h) /[p(e) \cdot p(h)] & \text { otherwise, }\end{cases} \\
R_{\text {norm }}(e, h) & = \begin{cases}R(e, h) /[p(\neg h) / p(h)] & \text { if } p(h \mid e) \geq p(h) \\
R(e, h) & \text { otherwise, }\end{cases} \\
G_{\text {norm }}(e, h) & = \begin{cases}G(e, h) & \text { if } p(h \mid e) \geq p(h) \\
G(e, h) /[p(h) / p(\neg h)] & \text { otherwise. }\end{cases}
\end{aligned}
$$

For our present purposes, two facts deserve emphasis. First of all, standard probability calculus yields that

$$
D_{\text {norm }}=S_{\text {norm }}=M_{\text {norm }}=N_{\text {norm }}=C_{\text {norm }}=R_{\text {norm }}=G_{\text {norm }} .
$$

In fact, at this point, we have one single (new) Bayesian measure of confirmation. From now on, we will label it $Z$.

The second important fact is that $Z$ does fulfill principle $\left(\mathrm{Ex}_{1}\right)$ above. The main formal results reported in this section are summarized in the following theorem and demonstrated in its proof (see Appendix A):

Theorem 1. $D, S, M, N, C, R$, and $G$ (and all confirmation measures ordinally equivalent to any of these) are inconsistent with principle $\left(\mathrm{Ex}_{1}\right)$, whereas $L$ and $Z$ (and all confirmation measures ordinally equivalent to any of these) satisfy principle $\left(\mathrm{Ex}_{1}\right)$.

3. $Z$-Measures. By means of $Z$ it is possible to define a whole set (indeed, a continuum) of confirmation measures (we will call them " $Z$-measures") that we would like to propose as an account of evidential support within the Bayesian framework. $Z$-measures are defined as follows:

$$
Z_{\alpha}= \begin{cases}Z(e, h)^{\alpha} & \text { if } p(h \mid e) \geq p(h) \\ -|Z(e, h)|^{\alpha} & \text { otherwise, }\end{cases}
$$

where $\alpha$ is positive. By being all monotone transformations of the same 
quantity, all $Z$-measures are ordinally equivalent. $\alpha$ plays the role of a parameter controlling curvature (see Figure 1).

As shown by the figure, when $\alpha=1$, the graphical representation of $Z_{\alpha}$ is composed of a pair of straight lines; when $\alpha<1, Z_{\alpha}$ is an S-shaped function, indicating a higher sensitivity of confirmation (disconfirmation) to relatively small positive (negative) departures from the prior probability of $h$; finally, when $\alpha>1, Z_{\alpha}$ is an inverse S-shaped function, indicating a higher sensitivity of confirmation (disconfirmation) to relatively wide positive (negative) departures from the prior probability of $h .^{5}$

Theorem 1 above immediately implies that all $Z$-measures fulfill principle $\left(\mathrm{Ex}_{1}\right)$. Here are some further desirable properties of $Z$-measures (which are not, however, sufficient to establish a better adequacy of $Z$ measures as compared to all their major competitors):

$\left(\mathrm{P}_{1}\right)$ If $p\left(h \mid e_{1}\right)>p\left(h \mid e_{2}\right)$, then $Z_{\alpha}\left(e_{1}, h\right)>Z_{\alpha}\left(e_{2}, h\right)$ (a property involved in the Bayesian solution of the "ravens paradox" provided by Horwich [1982]).

$\left(\mathrm{P}_{2}\right)$ If $h_{1} \vDash e, h_{2} \vDash e$, and $p\left(h_{1}\right)>p\left(h_{2}\right)$, then $Z_{\alpha}\left(e, h_{1}\right)>Z_{\alpha}\left(e, h_{2}\right)$ (a property involved in the Bayesian solution of the "grue paradox" provided by Sober [1994]).

$\left(\mathrm{P}_{3}\right)$ If $e$ confirms $h$ and $x$ is an "irrelevant conjunct" to $h$ with respect to $e$ (i.e., $p(e \mid h)=p(e \mid h \& x)$ ), then $Z_{\alpha}(e, h)>Z_{\alpha}(e, h \& x)$ (a property involved in the Bayesian solution of the paradox of "irrelevant conjunction" provided by Hawthorne and Fitelson [2004]).

$\left(\mathrm{P}_{4}\right)$ If $p\left(e \mid h_{1}\right)>\left(e \mid h_{2}\right)$ and $p\left(e \mid \neg h_{1}\right)<\left(e \mid \neg h_{2}\right)$, then $c\left(e, h_{1}\right)>c\left(e, h_{2}\right)$ (a principle known as the "weak law of likelihood" or "weak likelihood principle," which arguably "must be an integral part of any

5. Here we will not discuss the plausibility of various different values of $\alpha$. Notice, however, that on a descriptive level this free parameter might be subject to empirical investigation in the spirit of the experimental inquiry reported in the final section of the paper. (We are indebted to an anonymous referee for raising this point.) 

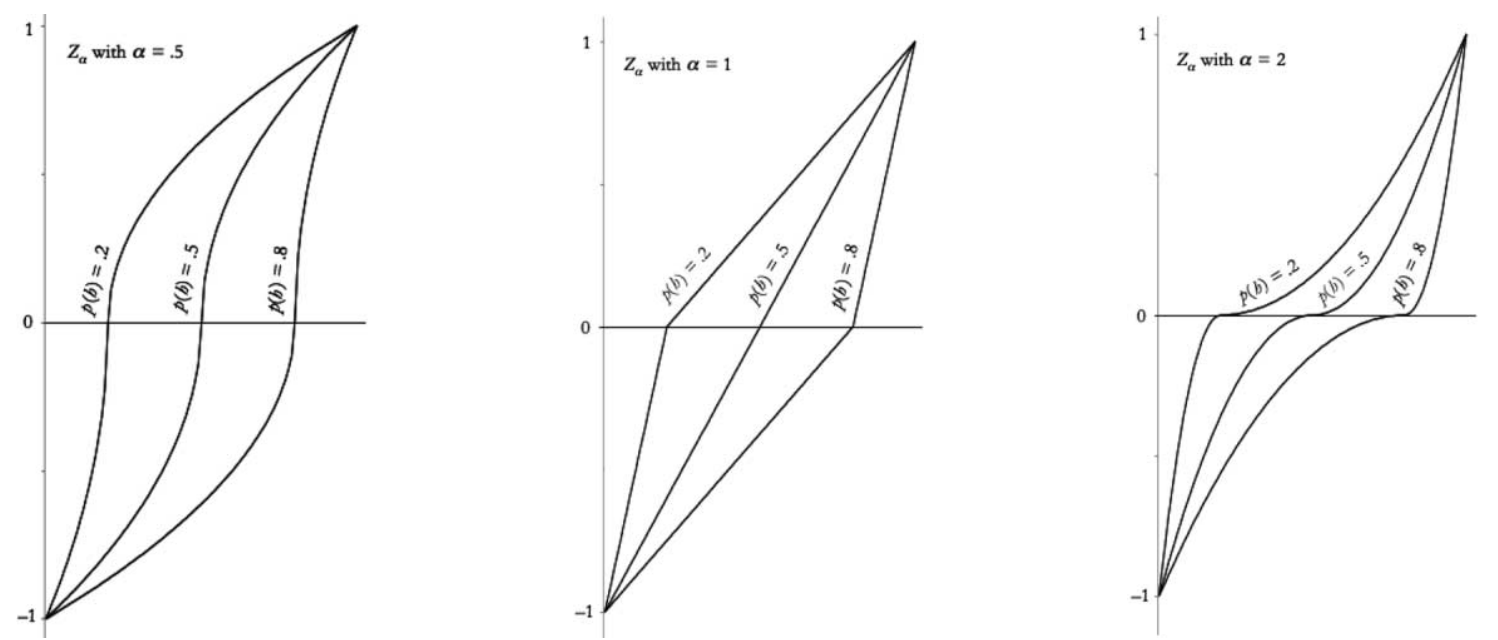

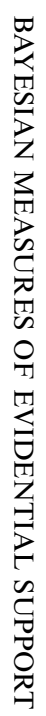

Figure 1. Graphical representations of Z-measures with different values of $\alpha$. On the $x$-axis lie posterior probability values of $h$. 
account of evidential relevance that deserves the title "Bayesian" [Joyce 2004]). ${ }^{6}$

4. Symmetries. Eells and Fitelson (2002) have recently proposed to narrow down the set of adequate Bayesian candidates for quantifying confirmation by considering a series of "symmetries and asymmetries." We will now present an extended and systematic treatment of this issue.

Consider a set of sentences $\Gamma$, closed under negation and conjunction, on which a probability function $p$ is defined. ${ }^{7}$ In what follows, by a "symmetry" we will mean a function $\sigma$ from $\Gamma \otimes \Gamma$ into $\Gamma \otimes \Gamma$ such that $\sigma(e$, $h)$ is obtained from $(e, h)$ by applying the negation operator $(\neg)$ to either $e$ or $h$ (or both) and/or by inverting them. On the whole, there are seven such symmetry functions:

$$
\begin{aligned}
E(e, h) & =(\neg e, h), \\
H(e, h) & =(e, \neg h), \\
I(e, h) & =(h, e), \\
E H(e, h) & =(\neg e, \neg h), \\
E I(e, h) & =(h, \neg e), \\
H I(e, h) & =(\neg h, e), \\
E H I(e, h) & =(\neg h, \neg e) .
\end{aligned}
$$

6. Interestingly, Fitelson (2006) has argued in favor of $L$ (and measures ordinally equivalent to $L$ ) by noticing its "historical uniqueness" (up to ordinal equivalence) in fulfilling his "logicality" requirement (see note 4 ) as well as property $\left(\mathrm{P}_{1}\right)$, while dismissing attempts to prove the mathematical uniqueness of $L$ (up to ordinal equivalence) because committing "continuity assumptions" concerning the probability function $p$ would be involved. (By the way, Fitelson is also aware that $L$ and ordinally equivalent measures enjoy property $\left(\mathrm{P}_{2}\right)$ [see Fitelson 2001,18 ] and property $\left(\mathrm{P}_{4}\right)$ [see Fitelson 2007]; also, Hawthorne and Fitelson [2004] have proved that such measures enjoy $\left(\mathbf{P}_{3}\right)$.) Now, it is an immediate corollary of the main formal result presented in this paper (see Theorem 2 below) that $Z$-measures are not ordinally equivalent to $L$ (nor, for that matter, to any other measure in Table 1). Thus, it seems that the definition of $Z$ measures (which are consistent with continuity assumptions concerning $p$ and which also demonstrably enjoy Fitelson's logicality) provides an existence proof that the uniqueness of $L$ (up to ordinal equivalence) is indeed just historical.

7. Again, strictly speaking, in order to exclude some degenerating cases in the analysis to follow, a few restrictions on $\Gamma$ and $p$ must be specified: beyond the regularity of $p$ and the contingency of $e$ and $h$ (see note 2), it is assumed that there exist $e, h \in \Gamma$ such that $p(e) \neq p(h)$ and $p(e \& h) \neq p(e) \cdot p(h)$ (i.e., that $e$ and $h$ are not probabilistically independent). 
Here, $E$ stands for "(negation of the) evidence," $H$ for "(negation of the) hypothesis," and $I$ for "inversion (of evidence and hypothesis)."

Now let $s$ (for "sign") be a function from $\Gamma \otimes \Gamma$ into $\{-1,0,1\}$, defined as follows: $s(e, h)=1$ if $p(h \mid e)>p(h) ; s(e, h)=0$ if $p(h \mid e)=p(h)$; and $s(e, h)=-1$ if $p(h \mid e)<p(h)$.

Function $s$ allows a distinction between convergent and divergent symmetries: a symmetry $\sigma$ is convergent iff $(e, h)$ and $\sigma(e, h)$ have the same sign, that is, iff, for any $e, h \in \Gamma, s(e, h)=s[\sigma(e, h)]$; a symmetry $\sigma$ is divergent iff $(e, h)$ and $\sigma(e, h)$ have opposite signs, that is, iff, for any $e, h \in \Gamma, s(e, h)=-s[\sigma(e, h)]$. It can easily be proved that $H, E, H I$, and $E I$ are divergent symmetries, whereas $I, E H$, and $E H I$ are convergent.

By means of $s$, we will also state the following definition:

Definition. $c$ mirrors a symmetry $\sigma$ in case of confirmation [disconfirmation] iff, for any $e, h \in \Gamma$ such that $s(e, h)=1[-1], s(e$, $h) \cdot c(e, h)=s(\sigma(e, h)) \cdot c(\sigma(e, h))$.

When a confirmation measure $c$ mirrors a symmetry $\sigma$ in both cases (confirmation and disconfirmation), then we will simply say that $c$ mirrors $\sigma$. (Notice that any measure $c$ trivially mirrors any symmetry $\sigma$ in case of neutrality, i.e., when $c(e, h)=0$.)

The conditions on which $c$ mirrors any particular symmetry $\sigma$ can now be stated as follows:

Divergent Symmetries.

$$
\begin{aligned}
& c(e, h)=-c(E(e, h))=-c(\neg e, h), \\
& c(e, h)=-c(H(e, h))=-c(e, \neg h), \\
& c(e, h)=-c(E I(e, h))=-c(h, \neg e), \\
& c(e, h)=-c(H I(e, h))=-c(\neg h, e) .
\end{aligned}
$$

\section{Convergent Symmetries.}

$$
\begin{aligned}
& c(e, h)=c(I(e, h))=c(h, e), \\
& c(e, h)=c(E H(e, h))=c(\neg e, \neg h), \\
& c(e, h)=c(E H I(e, h))=c(\neg h, \neg e) .
\end{aligned}
$$

5. A New Set of Adequacy Requirements. The point of Eells and Fitelson (2002) - whose discussion is in fact limited to $E, H, I$, and $E H^{8}$ - is that the appeal of $c$ mirroring any particular symmetry may be assessed by

8. These are precisely the symmetries originally discussed by Carnap ([1950] 1962 , Section 67). 
some rather simple and intuitive examples, which different measures of confirmation may or may not match.

To illustrate, take $E$ : Should it be the case that $c(e, h)=-c(\neg e, h)$ ?

Consider a standard deck of cards. It seems that having drawn a jack card confirms that the card is a face card to a greater extent than having drawn a card that is not a jack disconfirms the same hypothesis. The reason is that in the former case the available evidence conclusively establishes the hypothesis, whereas in the latter case the available evidence does not conclusively refute the hypothesis. Conversely, having drawn an ace card disconfirms that the card is a face card to a greater extent than having drawn a card that is not an ace confirms the same hypothesis. On the basis of similar examples, Eells and Fitelson (2002) argue that an acceptable Bayesian measure of confirmation should not mirror symmetry E.

Is there any general and simple principle to determine how a measure of confirmation should behave as far as all symmetries are concerned? We think there is one, which, just as $\left(\mathrm{Ex}_{1}\right)$ above (see Section 2), builds on the traditional view of inductive logic as an "extension" of classical deductive logic. Once again, such a principle may be stated by means of function $v$, defined on the basis of classical deductive logic (see Section 2):

$\left(\mathrm{Ex}_{2}\right) c$ mirrors $\sigma$ in case of confirmation [disconfirmation] iff $v$ mirrors $\sigma$ in case of confirmation [disconfirmation].

We shall see shortly why the distinction between the two cases (confirmation vs. disconfirmation) is important. For now, notice that $\left(\mathrm{Ex}_{2}\right)$ is a rather strong condition and, as we shall also see, a quite powerful theoretical tool. We don't know of any explicit and general treatment of it. However, it backs up several arguments circulating in inductive logic and in Bayesian confirmation theory in particular. For the moment, we will posit $\left(\mathrm{Ex}_{2}\right)$ as a legitimate guideline for the discussion of the symmetries that an adequate Bayesian measure of confirmation should or should not mirror. In what follows, we will try to establish consistency with $\left(\mathrm{Ex}_{2}\right)$ as a compelling desideratum through a detailed analysis of its consequences.

Resort to principle $\left(\mathrm{Ex}_{2}\right)$ may seem more adequate to identify the symmetries not to be mirrored (see, e.g., the jack and the ace examples above) rather than for the selection of those to be mirrored by $c$. However, the principle can be usefully applied in the latter sense as well, for instance as far as $H$ is concerned. Indeed, from $\left(\mathrm{Ex}_{2}\right)$ it follows that

$$
c(e, h)=-c(e, \neg h) .
$$

In the absence of compelling counterexamples (which we were not able 
to devise), the equality above can be presented as a plausible extension of the fact that $e$ implies $h$ (i.e., $e \vDash h$ ) iff $e$ refutes $\neg h$ (i.e., $e \vDash \neg \neg h$ ). ${ }^{9}$

Now consider symmetry $I$, that is, the following equality:

$$
c(e, h)=c(h, e)
$$

Should we require that a confirmation measure classify inversely symmetric arguments as equally strong (recall that the inverse symmetry function is convergent), that is, that it mirror $I$ ? Eells and Fitelson (2002) argue that we should not, but interestingly in this connection they explicitly resort only to counterexamples involving pairs of confirmatory arguments. This is noteworthy, for consider again the ace example above: in contrast to the jack case, we see as intuitively compelling that having drawn an ace card does disconfirm its being a face card to the same extent that having drawn a face card disconfirms its being an ace. Thus, we concur with Eells and Fitelson in finding unattractive the above equality in case of confirmation but not necessarily in case of disconfirmation as well. Indeed, $c$ mirroring $I$ in case of disconfirmation (but not in case of confirmation) also follows from $\left(\mathrm{Ex}_{2}\right)$ and, in the absence of compelling counterexamples (which we were not able to devise), this seems a plausible extension of the theorem of deductive logic according to which $e \vDash \neg h$ iff $h \vDash \neg e$, that is, a plausible inductive-theoretic counterpart of the commutative (or "inversely symmetric") nature of logical inconsistency.

Generalizing this line of argument, one can show that principle $\left(\mathrm{Ex}_{2}\right)$ yields a definite answer for any symmetry both in case of confirmation and in case of disconfirmation. Table 3 reports the outcomes of such analysis, illustrating in any single case either the relevant parallelism with an analogous state of affairs whithin deductive logic or relevant counterexamples.

6. Why Z-Measures Are Theoretically Appealing. We claim that $Z$-measures should be of interest for any Bayesian confirmation theorist who finds principle $\left(\mathrm{Ex}_{2}\right)$ compelling for the plain fact that such measures are all consistent with $\left(\mathrm{Ex}_{2}\right)$ whereas, to our knowledge, no alternative measure proposed so far is. The proof of the following theorem (see Appendix

9. Interestingly, Kemeny and Oppenheim (1952) derived their proposal of measure $L$ from a set of requirements that includes mirroring symmetry $H$. They posit such a requirement as "natural" and seem to presuppose $\left(\mathrm{Ex}_{2}\right)$ in their argument. Eells and Fitelson (2002) take a similar position. 
TABle 3. The Whole Set of Consequences of $\left(\mathrm{Ex}_{2}\right)$

\begin{tabular}{|c|c|c|c|c|}
\hline \multirow[b]{3}{*}{ E } & \multicolumn{2}{|c|}{ In Case of Confirmation } & \multicolumn{2}{|c|}{ In Case of Disconfirmation } \\
\hline & \multicolumn{4}{|c|}{ Divergent Symmetries } \\
\hline & No & & No & \\
\hline & $\begin{array}{l}\text { For some confirmation }(e, h) \\
\qquad(e, h) \neq-c(\neg e, h)\end{array}$ & $\begin{array}{c}\text { e.g., } c(\text { jack, face })>-c \text { (not- } \\
\text { jack, face })\end{array}$ & $\begin{array}{l}\text { For some disconfirmation }(e, h) \\
\qquad c(e, h) \neq-c(\neg e, h)\end{array}$ & $\begin{array}{c}\text { e.g., } c \text { (ace, face })<-c \text { (not- } \\
\text { ace, face })\end{array}$ \\
\hline$H$ & For any confirmation $(e, h)$ & $e$ implies $h$ iff $e$ refutes $\neg h$ & $\begin{array}{c}\text { Yes } \\
\text { For any disconfirmation }(e, h)\end{array}$ & $e$ refutes $h$ iff $e$ implies $\neg h$ \\
\hline EI & $\begin{array}{c}c(e, h)=-c(e, \neg h) \\
\text { No }\end{array}$ & & $\begin{array}{c}c(e, h) \underset{\text { Yes }}{=}-c(e, \neg h) \\
\text { (a) }\end{array}$ & \\
\hline \multirow{3}{*}{$H I$} & $\begin{array}{l}\text { For some confirmation }(e, h) \\
c(e, h) \neq-c(h, \neg e)\end{array}$ & $\begin{array}{l}\text { e.g., } c(\text { jack, face })> \\
-c(\text { face, not-jack })\end{array}$ & $\begin{array}{c}\text { For any disconfirmation }(e, h) \\
c(e, h)=-c(h, \neg e)\end{array}$ & $e$ refutes $h$ iff $h$ implies $\neg e$ \\
\hline & $\begin{array}{c}\text { Yes } \\
\text { For any confirmation }(e, h) \\
c(e, h)=-c(\neg h, e)\end{array}$ & $e$ implies $h$ iff $\neg h$ refutes $e$ & $\begin{array}{c}\text { No } \\
\text { For some disconfirmation }(e, h) \\
c(e, h) \neq-c(\neg h, e)\end{array}$ & $\begin{array}{c}\text { e.g., } c \text { (ace, face })<-c \text { (not- } \\
\text { face, ace })\end{array}$ \\
\hline & \multicolumn{4}{|c|}{ Convergent Symmetries } \\
\hline I & No & & Yes & \\
\hline & $\begin{array}{l}\text { For some confirmation }(e, h) \\
\qquad c(e, h) \neq c(h, e)\end{array}$ & 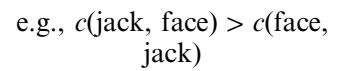 & $\begin{array}{c}\text { For any disconfirmation }(e, h) \\
\qquad(e, h)=c(h, e)\end{array}$ & $e$ refutes $h$ iff $h$ refutes $e$ \\
\hline$E H$ & & & No & \\
\hline & $\begin{array}{l}\text { For some confirmation }(e, h) \\
\qquad(e, h) \neq c(\neg e, \neg h)\end{array}$ & $\begin{array}{l}\text { e.g., } c(\text { jack, face })>c(\text { not- } \\
\text { jack, not-face })\end{array}$ & $\begin{array}{l}\text { For some disconfirmation }(e, h) \\
\qquad c(e, h) \neq c(\neg e, \neg h)\end{array}$ & $\begin{array}{c}\text { e.g., } c \text { (ace, face })<c(\text { not- } \\
\text { ace, not-face })\end{array}$ \\
\hline$E H I$ & & & No & \\
\hline & $\begin{array}{c}\text { For any confirmation }(e, h) \\
\quad c(e, h)=c(\neg h, \neg e)\end{array}$ & $\begin{array}{c}e \text { implies } h \text { iff } \neg h \text { implies } \\
\neg e\end{array}$ & $\begin{array}{l}\text { For some disconfirmation }(e, h) \\
\qquad c(e, h) \neq c(\neg h, \neg e)\end{array}$ & $\begin{array}{l}\text { e.g., } c(\text { ace, face })<c(\text { not- } \\
\text { face, not-ace })\end{array}$ \\
\hline
\end{tabular}


B), which is the main formal result of the present work, illustrates the point. ${ }^{10}$

Theorem 2. $Z$-measures satisfy all the consequences of $\left(\mathrm{Ex}_{2}\right)$, whereas $D, S, M, N, C, R, G$, and $L$ (and all confirmation measures ordinally equivalent to any of these) are inconsistent with $\left(\mathrm{Ex}_{2}\right) .{ }^{11}$

The fact that measure $L$ does fulfill principle $\left(\mathrm{Ex}_{1}\right)$ but not $\left(\mathrm{Ex}_{2}\right)$ shows that the former does not imply the latter. Notably, it is also not the case that $\left(\mathrm{Ex}_{2}\right)$ implies $\left(\mathrm{Ex}_{1}\right) \cdot{ }^{12}$ Thus both principles are complementary but independent constraints stemming from the same idea of confirmation theory as an extension of deductive logic. Arguably, however, one may still cast doubts on this strict parallelism and question why, after all, we should want a formal account of inductive reasoning to be consistent with $\left(\mathrm{Ex}_{2}\right)$.

Let's take symmetry $I$ as an example about which such doubts may typically be raised. Suppose that you must rely on two generally accurate but fallible devices $A_{1}$ and $A_{2}$ in order to obtain information about a playing card. $A_{1}$ works as follows: it classifies a submitted card as a jack or a not-jack and reports the outcome with an accuracy rate of $95 \%$ (not depending on whether the card is or is not, in fact, a jack). $A_{2}$ works as follows: it classifies a submitted card as a face or not-face and reports the outcome, again with an accuracy rate of $95 \%$ (not depending on whether the card is or is not, in fact, a face). Now suppose that a card is drawn from a standard deck by a genuinely random process and submitted to both $A_{1}$ and $A_{2}$. Let $e$ be " $A_{1}$ reports that it is a jack card" and $h$ be " $A_{2}$ reports that it is a face card." On reflection, it seems clear to us that $e$ confirms $h$ more than $h$ confirms $e$, even if none is strictly implied by the other. (If this example sounds unbearably artificial, let $A_{1}$ and

10. Here it is appropriate to mention the only previous occurrence of $Z$-measures that we have been able to detect in the literature. Rescher (1958) noticed measure $Z$ (i.e., $Z_{\alpha}$ with $\alpha=1$ ) and even displayed its graphical representation. However, on the basis of a set of adequacy requirements that did not include consistency with $\left(\mathrm{Ex}_{2}\right)$, Rescher eventually identified $Z(e, h) \cdot p(e)$ as the most adequate explicatum for the degree of evidential support. The difference may seem minor but is not. As a matter of fact, it can be shown that Rescher's measure does not share many of the properties of $Z$ measures that have been discussed here and, in particular, is not consistent with $\left(\mathrm{Ex}_{2}\right)$.

11. Interestingly, ordinal equivalence to $Z$-measures is not a sufficient condition for a confirmation measure to be consistent with $\left(\mathrm{Ex}_{2}\right)$. To see why, consider a measure defined as follows: $[Z(e, h)]^{\alpha}$ if $p(h \mid e) \geq p(h)$ and $-|Z(e, h)|^{\beta}$ otherwise (where both $\alpha$ and $\beta$ are positive). Whenever $\alpha \neq \beta$, such a measure is ordinally equivalent to all $Z$ measures, but it also violates $\left(\mathrm{Ex}_{2}\right)$ (for instance, it does not mirror symmetry $H$ ).

12. In fact, Bayesian measures of confirmation can be defined that satisfy $\left(\mathrm{Ex}_{2}\right)$ but not $\left(\mathrm{Ex}_{1}\right)$, for instance, $Z(e, h) /|C(e, h)|$. 
$A_{2}$ be two medical tests for two diagnostic hypotheses such that the former implies the latter.)

But now consider a third device $A_{3}$ such that it classifies a card as an ace or a not-ace, again with an accuracy rate of $95 \%$ (not depending on whether the card is or is not, in fact, an ace). Suppose that a card is selected by a new random extraction and submitted to both $A_{3}$ and $A_{2}$. Let $e^{*}$ be " $A_{3}$ reports that it is an ace" and $h$, once again, " $A_{2}$ reports that it is a face card." Here, we see no reason why the two arguments $\left(e^{*}, h\right)$ and $\left(h, e^{*}\right)$ should have a different (negative) strength. Sentences $e^{*}$ and $h$ are just "almost" incompatible; and, so it seems, that is all. (If this example sounds unbearably artificial, now let $A_{3}$ and $A_{2}$ be two medical tests for two mutually exclusive diagnostic hypotheses.)

The problem is that, unlike $Z$-measures, all the competitors violating $\left(\mathrm{Ex}_{2}\right)$ also violate intuition here by either ranking $(e, h)$ as an equally strong confirmatory argument as $(h, e)^{13}$ or ranking one between $\left(e^{*}, h\right)$ and $\left(h, e^{*}\right)$ as a significantly stronger disconfirmatory argument than the other (without even agreeing on which one is the strongest!). ${ }^{14}$

Similar examples may be conceived for any row in Table 2 to underpin the plausibility of $\left(\mathrm{Ex}_{2}\right)$ and therefore of $Z$-measures as a theoretically appropriate basis to assess inductive strength.

7. An Empirical Test of Competing Measures. Formal theories of reasoning may serve various purposes. So far, we have been concerned with the elucidation of a central epistemological concept (confirmation) on the basis of a set of intuitively plausible and explicitly stated constraints. Whether or not a theoretically sound measure of confirmation may also be employed to accurately describe (and predict) human judgment and behavior is a distinguished question - a very intriguing one, to which we will now turn. Our purpose is to extend the assessment of the potentiality of the proposed approach to the empirical domain of the psychology of reasoning and cognition, which has often widely profited from the comparison between actual human performance and compelling formal theories such as probability theory or expected utility theory.

In a previous work (Tentori et al. 2007), an experiment has been presented aiming at an empirical test of some of the alternative Bayesian measures of confirmation. For each of 26 participants, an urn was randomly and covertly selected out of $A$ (30 black balls plus 10 white balls) and $B$ ( 15 black balls plus 25 white balls). Participants were then asked

13. In fact, it will be the case that $c(e, h)=c(h, e)$ if $c$ stands for either $C$ or $R$ (see Appendix B).

14. In fact, it will be the case that $c\left(e^{*}, h\right)<c\left(h, e^{*}\right)$ if $c$ stands for $D, S, G$, or $L$, and $c\left(e^{*}, h\right)>c\left(h, e^{*}\right)$ if $c$ stands for $M$ or $N$ (computational details are omitted). 
TABle 4. Average Correlations for Competing CONFIRMATION MEASURES.

\begin{tabular}{|c|c|c|}
\hline \multirow{2}{*}{$\begin{array}{l}\text { Measure } \\
\text { (Predictor) }\end{array}$} & \multicolumn{2}{|c|}{$\begin{array}{l}\text { Average Correlation with Participants' } \\
\text { Confirmation Judgments for the } \\
\text { Hypothesis: }\end{array}$} \\
\hline & Urn $A$ Selected & Urn $B$ Selected \\
\hline$Z$ & $.756^{*}$ & $.775^{*}$ \\
\hline$L$ & $.740^{*}$ & $.754 *$ \\
\hline$N$ & $.730 *$ & $.745^{*}$ \\
\hline$M$ & $.628^{\dagger}$ & .588 \\
\hline$G$ & .549 & .631 \\
\hline$R$ & .619 & .557 \\
\hline$S$ & .594 & .613 \\
\hline$C$ & .586 & .605 \\
\hline$D$ & .573 & .589 \\
\hline$p(A[B] \mid e)$ & .488 & .508 \\
\hline \multicolumn{3}{|c|}{$\begin{array}{l}\text { NOTE.-Reported values are the average of } 26 \text { correlations (one per participant) } \\
\text { between the confirmation judgments predicted by each measure (on the basis of the } \\
\text { randomness of the initial selection, the composition of the urns, and the outcomes } \\
\text { of previous draws) and the confirmation judgments expressed by each participant. } \\
\text { Each correlation involved } 10 \text { observations. } p(A[B] \mid e) \text { denotes } p(A \mid e) \text { or } p(B \mid e) \text { as } \\
\text { appropriate. Comparisons by paired } t \text {-test with the average correlation for } \\
p(A[B] \mid e):{ }^{*} p<.001 ;{ }^{t} p=.06 \text {. }\end{array}$} \\
\hline
\end{tabular}

to judge the quantitative impact of each outcome of a series of 10 random extractions without replacement on the two complementary hypotheses that $A$ versus $B$ had been selected (see Tentori et al. [2007] for a detailed description of the procedure). The experiment showed that, when predictions about confirmation judgments were computed from the randomness of the initial selection, the composition of the urns, and the outcomes of previous draws, measure $L$ outperformed several competitors (including $D, N$, and $C$ ), yielding a reliably higher average correlation with the judged impacts on both hypotheses $A$ and $B$ provided by the participants. In what follows we will test all the competing confirmation measures listed in Table 1 , along with the simplest $Z$-measure (i.e., with $\alpha=1$ ), against the same data.

As Table 4 shows, $Z$ is the most descriptively accurate measure in this experimental setting. (The table also reports a reliable superiority of the most accurate measures in comparison with posterior probability, taken as a predictor of confirmation judgments. Notice that this indicates that the participants appropriately distinguished posteriors from evidential impact.)

Consistent with these results, Table 5 shows that, by a paired $t$-test, $Z$ outperforms all competitors in predicting judged impact on both hypotheses $A$ and $B$, with only one major exception (the lack of a statistically reliable difference from $L$ for hypothesis $A$ ). Moreover, a simple nonparametric analysis consisting in counting the subjects (out of 26) for 
TABle 5. Comparison of $Z$ With Other Confirmation Measures.

\begin{tabular}{|c|c|c|c|c|c|c|c|c|}
\hline & $L$ & $N$ & $M$ & $G$ & $R$ & $S$ & $C$ & $D$ \\
\hline & \multicolumn{8}{|c|}{ Hypothesis: Urn $A$ Selected } \\
\hline \multirow[t]{2}{*}{$Z$} & $\begin{array}{c}t=1,44 \\
\text { n.s. } \\
18\end{array}$ & $\begin{array}{c}t=1,92 \\
p=.07 \\
18\end{array}$ & $\begin{array}{c}t=2,87 \\
p=.008 \\
18\end{array}$ & $\begin{array}{c}t=4,52 \\
p=.0001 \\
22\end{array}$ & $\begin{array}{c}t=3,25 \\
p=.003 \\
21\end{array}$ & $\begin{array}{c}t=3,13 \\
p=.004 \\
21\end{array}$ & $\begin{array}{c}t=3,25 \\
p=.003 \\
21\end{array}$ & $\begin{array}{c}T=3,61 \\
p=.001 \\
21\end{array}$ \\
\hline & \multicolumn{8}{|c|}{ Hypothesis: Urn $B$ Selected } \\
\hline$Z$ & $\begin{array}{c}t=2,02 \\
p=.05 \\
18\end{array}$ & $\begin{array}{c}t=2,31 \\
p=.03 \\
18\end{array}$ & $\begin{array}{c}t=3,50 \\
p=.002 \\
22\end{array}$ & $\begin{array}{c}t=3,51 \\
p=.002 \\
24\end{array}$ & $\begin{array}{c}t=4,01 \\
p=.0005 \\
23\end{array}$ & $\begin{array}{c}t=2,84 \\
p=.009 \\
19\end{array}$ & $\begin{array}{c}t=2,96 \\
p=.007 \\
19\end{array}$ & $\begin{array}{c}t=3,28 \\
p=.003 \\
21\end{array}$ \\
\hline
\end{tabular}

NoTE.-Each higher cell reports a paired $t$-test between the correlations obtained from the measure in the associated column and from measure $Z$. Each lower cell shows the number of participants (out of 26 ) for whom $Z$ predicted better than the rival measure at the top of the column. 
whom $Z$ predicted better than each rival measure shows that $Z$ is generally a better predictor in the majority of cases (see Table 5).

These results suggest that the virtues of $Z$-measures might not be confined to the level of epistemological analysis, but extend to the descriptive dimension of the psychology of confirmation.

We are not claiming that the probabilistic computations involved in the definition of Bayesian confirmation measures should be taken literally as models of the cognitive processes leading to confirmation judgments. We are well aware that, when judging probabilities, subjects often depart from the Bayesian prescriptions (Kahneman, Slovic, and Tversky 1982) and conform to them only under specific conditions (Girotto and Gonzalez 2001). Precisely for this reason, it is noticeable that a quantitative Bayesian account of evidential impact can reach a promising predictive accuracy relative to naïve judgments of confirmation. Whatever the routes by which confirmation judgments are elaborated by nonexpert subjects, the experiment illustrates that some salient features of such judgments may be captured, to a significant degree, by a theoretically sound account of confirmation. In Marr's (1982) terms, such an account might work as an effective, although approximated, computational-level model, compatible with more "realistic" process-level descriptions of the cognitive bases of ordinary inductive reasoning.

In any event, more research is needed to assess the stability and the consequences of the present results. In a touchstone study in the psychology of inductive reasoning with statements involving familiar biological categories and "blank" biological predicates (such as "robins use serotonin as a neurotransmitter"), Osherson et al. (1990) documented that inversely symmetric confirmatory arguments are not generally judged as equally strong, consistent with principle $\left(\mathrm{Ex}_{2}\right)$ (see Table 3$)$. Further studies aiming at the empirical test of various symmetries and asymmetries might provide evidence about substantial qualitative phenomena concerning inductive reasoning and play a crucial role in assessing the descriptive reliability of competing measures of confirmation outside the context of our urn-based experiment.

In our opinion, the discussion presented here suggests that the interaction between the epistemological and the cognitive dimension might be fruitful in this field of inquiry as it has been in many other areas of the study of reasoning. 


\section{Appendix A}

Theorem 1. $D, S, M, N, C, R$, and $G$ (and all confirmation measures ordinally equivalent to any of these) are inconsistent with principle $\left(\mathrm{Ex}_{1}\right)$, whereas $L$ and $Z$ (and all confirmation measures ordinally equivalent to any of these) satisfy principle $\left(\mathrm{Ex}_{1}\right)$.

Proof

Simple probability models prove the first part of the theorem.

Suppose that $x, y$, and $z$ are pairwise logically incompatible, that $p(x)+p(y)+p(z)=1$, and that $p(z)>.5$. (For instance, a fair die is tossed: $x=1, y=2, z=$ strictly greater than 2 .) Then let it be the case that $e_{1}=y, e_{2}=(x \vee y), h_{1}=(y \vee z)$, and $h_{2}=x$. Notice that $e_{1} \vDash h_{1}$ while $e_{2} \neq h_{2}$; therefore, $v\left(e_{1}, h_{1}\right)>v\left(e_{2}, h_{2}\right)$. Moreover, $e_{1} \vDash \neg\left(\neg h_{1}\right)$ while $e_{2} \# \neg\left(\neg h_{2}\right)$; therefore, $v\left(e_{1}, \neg h_{1}\right)<v\left(e_{2}, \neg h_{2}\right)$.

Now $D\left(e_{1}, h 1\right)=p(x)$, while $D\left(e_{2}, h_{2}\right)=\{p(x) /[p(x)+p(y)]\}-p(x)$. It follows that $D\left(e_{1}, h_{1}\right)<D\left(e_{2}, h_{2}\right)$ iff $[p(x)+p(y)]<1 / 2$, and the latter is (by hypothesis) the case.

Since $S(e, h)=D(e, h) / p(\neg e), S\left(e_{1}, h_{1}\right)<S\left(e_{2}, h_{2}\right)$ immediately follows from $D\left(e_{1}, h_{1}\right)<D\left(e_{2}, h_{2}\right)$ and $p\left(\neg e_{1}\right)>p\left(\neg e_{2}\right)$.

Since $p\left(e_{1}\right)<p\left(e_{2}\right)$ and $p\left(h_{1}\right)>p\left(h_{2}\right)$, it follows that $p\left(e_{1}\right) / p\left(h_{1}\right)<$ $p\left(e_{2}\right) / p\left(h_{2}\right)$. And since $M(e, h)=D(e, h) \cdot[p(e) / p(h)]$ and $D\left(e_{1}, h_{1}\right)<$ $D\left(e_{2}, h_{2}\right)$, it follows that $M\left(e_{1}, h_{1}\right)<M\left(e_{2}, h_{2}\right)$.

Since $C(e, h)=D(e, h) \cdot p(e), p\left(e_{1}\right)<p\left(e_{2}\right)$, and $D\left(e_{1}, h_{1}\right)<D\left(e_{2}, h_{2}\right)$, it follows that $C\left(e_{1}, h_{1}\right)<C\left(e_{2}, h_{2}\right)$.

Since $h_{1}$ and $\neg h_{2}$ are equivalent, it follows that $p\left(h_{1}\right) \cdot p\left(\neg h_{1}\right)=$ $p\left(h_{2}\right) \cdot p\left(\neg h_{2}\right)$. Then, since $N(e, h)=C(e, h) /[p(h) \cdot p(\neg h)]$ and $C\left(e_{1}\right.$, $\left.h_{1}\right)<C\left(e_{2}, h_{2}\right)$, it follows that $N\left(e_{1}, h_{1}\right)<N\left(e_{2}, h_{2}\right)$.

Since $p\left(h_{1}\right)>p\left(h_{2}\right)$ and $R(e, h)=D(e, h) / p(h), R\left(e_{1}, h_{1}\right)<R\left(e_{2}, h_{2}\right)$ follows from $D\left(e_{1}, h_{1}\right)<D\left(e_{2}, h_{2}\right)$.

Finally, since $G(e, \neg h)=-R(e, h)$, from $R\left(e_{1}, h_{1}\right)<R\left(e_{2}, h_{2}\right)$ it follows that $G\left(e_{1}, \neg h_{1}\right)>G\left(e_{2}, \neg h_{2}\right)$.

As far as the second part of the theorem is concerned, notice that, if $v\left(e_{1}, h_{1}\right)>v\left(e_{2}, h_{2}\right)$, then (assuming that $p$ is regular) at least one of the following is true:

a. $p\left(h_{1} \mid e_{1}\right)=1$ and $p\left(h_{2} \mid e_{2}\right)<1$ (equivalently, $p\left(e_{1} \mid \neg h_{1}\right)=0$ and $\left.p\left(e_{2} \mid \neg h_{2}\right)>0\right)$.

b. $p\left(h_{1} \mid e_{1}\right)>0$ and $p\left(h_{2} \mid e_{2}\right)=0$ (equivalently, $p\left(e_{1} \mid h_{1}\right)>0$ and $\left.p\left(e_{2} \mid h_{2}\right)=0\right)$. 
Suppose that part a is true. Then $L\left(e_{1}, h_{1}\right)=p\left(e_{1} \mid h_{1}\right) / p\left(e_{1} \mid h_{1}\right)=1$ and $Z\left(e_{1}, h_{1}\right)=p\left(\neg h_{1}\right) / p\left(\neg h_{1}\right)=1$, whereas simple algebraic considerations show that $L\left(e_{2}, h_{2}\right)<1$ and $Z\left(e_{2}, h_{2}\right)<1$.

Now suppose that part $\mathrm{b}$ is true. Then $L\left(e_{2}, h_{2}\right)=-p\left(e_{2} \mid \neg h_{2}\right) / p\left(e_{2} \mid\right.$ $\left.\neg h_{2}\right)=-1$ and $Z\left(e_{2}, h_{2}\right)=-p\left(h_{2}\right) / p\left(h_{2}\right)=-1$, whereas simple algebraic considerations show that $L\left(e_{1}, h_{1}\right)>-1$ and $Z\left(e_{1}, h_{1}\right)>-1$.

Thus, if either part a or part $\mathrm{b}$ is true (which is always the case if $\left.v\left(e_{1}, h_{1}\right)>v\left(e_{2}, h_{2}\right)\right)$, then both $L\left(e_{1}, h_{1}\right)>L\left(e_{2}, h_{2}\right)$ and $Z\left(e_{1}, h_{1}\right)>Z\left(e_{2}\right.$, $\left.h_{2}\right)$.

\section{Appendix B}

Theorem 2. $Z$-measures satisfy all the consequences of $\left(\mathrm{Ex}_{2}\right)$, whereas $D, S, M, N, C, R, G$, and $L$ (and all confirmation measures ordinally equivalent to any of these) are inconsistent with $\left(\mathrm{Ex}_{2}\right)$.

\section{Proof}

In order to prove the theorem we will use three lemmas.

Lemma 1. Any confirmation measure $c$ satisfying (BC) also satisfies all the consequences of $\left(\mathrm{Ex}_{2}\right)$ iff $c$ mirrors $H$ and $c$ mirrors $I$ in case of confirmation but not in case of disconfirmation.

Proof. Trivially, if $c$ satisfies all the consequences of $\left(\mathrm{Ex}_{2}\right)$, then $c$ mirrors $H$ and $c$ mirrors $I$ in case of confirmation but not in case of disconfirmation.

On the other hand, suppose that $c$ does mirror $H$ and does mirror $I$ in case of confirmation but not in case of disconfirmation. Then, for any $e, h \in \Gamma$ such that $s(e, h)=1$,

$$
\begin{aligned}
c(e, h)=-c(e, \neg h) & {[\text { since } c \text { mirrors } H] } \\
-c(e, \neg h)=-c(\neg h, e) & {[\text { since } c \text { mirrors } I \text { in case of }} \\
& \text { disconfirmation }] \\
c(e, h)=-c(\neg h, e), & \text { therefore, } c \text { mirrors } H I \text { in case of } \\
& \text { confirmation and } E I \text { in case of } \\
& \text { disconfirmation }
\end{aligned}
$$




$$
\begin{array}{rlrl}
-c(\neg h, e) & =c(\neg h, \neg e) & & {[\text { since } c \text { mirrors } H]} \\
c(e, h)=c(\neg h, \neg e), & & \text { therefore, } c \text { mirrors } E H I \text { in case } \\
& \text { of confirmation. }
\end{array}
$$

Moreover, for some $e, h \in \Gamma$ such that $s(e, h)=1$,

$$
\begin{aligned}
c(e, h) \neq c(h, e) & {[\text { since } c \text { does not mirror } I} \\
& \text { in case of confirmation] } \\
c(h, e)=-c(h, \neg e) & {[\text { since } c \text { mirrors } H] } \\
-c(h, \neg e)=-c(\neg e, h) & {[\text { since } c \text { mirrors } I \text { in case of }} \\
& \text { disconfirmation] } \\
-c(\neg e, h)=c(\neg e, \neg h) & {[\text { since } c \text { mirrors } H] } \\
c(e, h)=-c(e, \neg h) & {[\text { since } c \text { mirrors } H] } \\
& \\
c(e, h) \neq-c(h, \neg e), & \text { therefore, } c \text { does not mirror } \\
& \text { EI in case of confirmation } \\
& \text { and does not mirror } H I \text { in } \\
& \text { case of disconfirmation; } \\
c(e, h) \neq-c(\neg e, h) & \\
c(e, h) \neq c(\neg e, \neg h) & \text { therefore, } c \text { does not mirror } E ; \\
c(e, \neg h) \neq c(h, \neg e) & \text { therefore, } c \text { does not mirror } E H ; \\
& \text { therefore, } c \text { does not mirror } E H I \\
& \text { in case of disconfirmation. }
\end{aligned}
$$

Lemma 2. $Z$ mirrors $H$ and $Z$ mirrors $I$ in case of confirmation but not in case of disconfirmation. 
Proof. $Z$ mirrors $H$ because, for any $e, h \in \Gamma$ such that $s(e, h)=1$,

$$
\begin{aligned}
Z(e, h) & =[p(h \mid e)-p(h)] / p(\neg h) \\
& =[p(\neg h)-p(\neg h \mid e)] / p(\neg h) \\
& =-[p(\neg h \mid e)-p(\neg h)] / p(\neg h) \\
& =-Z(e, \neg h) .
\end{aligned}
$$

Moreover, $Z$ mirrors $I$ in case of disconfirmation because, for any $e, h \in \Gamma$ such that $s(e, h)=-1$,

$$
\begin{aligned}
Z(e, h) & =[p(h \mid e)-p(h)] / p(h) \\
& =[p(h \mid e) / p(h)]-1 \\
& =[p(e \mid h) / p(e)]-1 \\
& =[p(e \mid h)-p(e)] / p(e) \\
& =Z(h, e) .
\end{aligned}
$$

Finally, $Z$ does not mirror $I$ in case of confirmation because, for any $e, h \in \Gamma$ such that $s(e, h)=1$, when we posit $Z(e, h)=Z(h, e)$, it follows that $p(h) / p(\neg h)=p(e) / p(\neg e)$ (computations are omitted), which implies that, whenever $p(h) \neq p(e), Z(e, h) \neq Z(h, e)$.

Lemma 3. If a confirmation measure $c$ satisfying (BC) is a function $f$ of $Z$ (i.e., $c(e, h)=f[Z(e, h)])$ such that $f$ is injective and $f(x)=$ $-f(-x)$, then $c$ also satisfies all the consequences of $\left(\mathrm{Ex}_{2}\right)$.

Proof. If $c$ is a function of $Z$, then, by definition, $Z(e, h)=Z(h, e)$ implies $c(e, h)=c(h, e)$. Therefore, since $Z$ mirrors $I$ in case of disconfirmation (Lemma 2), the same is true of $c$. Moreover, if $c$ is an injective function of $Z$, then by definition, $Z(e, h) \neq Z(h, e)$ implies $c(e, h) \neq c(h, e)$. Therefore, since $Z$ does not mirror $I$ in case of confirmation (Lemma 2), the same is true of $c$. Finally, if $c(e, h)=$ $f[Z(e, h)]$ and $f(x)=-f(-x)$, then $Z(e, h)=-Z(e, \neg h)$ implies $c(e$, $h)=-c(e, \neg h)$. Therefore, since $Z$ mirrors $H$ (Lemma 2), the same is true of $c$.

By Lemma 1, it follows that if $c$ is a function $f$ of $Z$ such that $f$ is injective and $f(x)=-f(-x)$, then $c$ satisfies all the consequences of $\left(\mathrm{Ex}_{2}\right)$.

To prove the theorem, we will now prove that 
a. any $Z$-measure is an injective function $f$ of $Z$ such that $f(x)=$ $-f(-x)$ and therefore, by Lemma 3 , satisfies all the consequences of $\left(\mathrm{Ex}_{2}\right)$;

b. $D, S, M, N, C, R, G$, and $L$ (and all measures of confirmation ordinally equivalent to any of these) are inconsistent with $\left(\mathrm{Ex}_{2}\right)$.

Proof of part $a$. The very definition of $Z$-measures shows that they are all functions of $Z$.

Notice that, by principle (BC), for any $e_{1}, e_{2}, h_{1}, h_{2} \in \Gamma$ such that $s\left(e_{1}, h_{1}\right)=1$ and $s\left(e_{2}, h_{2}\right)=-1, Z\left(e_{1}, h_{1}\right) \neq Z\left(e_{2}, h_{2}\right)$ and $Z_{\alpha}\left(e_{1}, h_{1}\right) \neq$ $Z_{\alpha}\left(e_{2}, h_{2}\right)$. Moreover, it immediately follows from the definition of $Z$ measures that, for any $e_{1}, e_{2}, h_{1}, h_{2} \in \Gamma$ such that $s\left(e_{1}, h_{1}\right)=s\left(e_{2}\right.$, $\left.h_{2}\right)=1, Z\left(e_{1}, h_{1}\right) \neq Z\left(e_{2}, h_{2}\right)$ implies $Z_{\alpha}\left(e_{1}, h_{1}\right) \neq Z_{\alpha}\left(e_{2}, h_{2}\right)$ and that, for any $e_{1}, e_{2}, h_{1}, h_{2} \in \Gamma$ such that $s\left(e_{1}, h_{1}\right)=s\left(e_{2}, h_{2}\right)=-1, Z\left(e_{1}\right.$, $\left.h_{1}\right) \neq Z\left(e_{2}, h_{2}\right)$ implies $Z_{\alpha}\left(e_{1}, h_{1}\right) \neq Z_{\alpha}\left(e_{2}, h_{2}\right)$. This shows that any $Z$ measure is an injective function of $Z$.

Finally, let it be the case that $Z\left(e_{1}, h_{1}\right)=x$ and $Z\left(e_{2}, h_{2}\right)=-x$. It is now sufficient to notice that $[x]^{\alpha}=|-x|^{\alpha}=-\left[-|-x|^{\alpha}\right]$. This shows that any $Z$-measure is a function $f$ of $Z$ such that $f(x)=-f(-x)$, and completes the proof of part a.

Proof of part $b$. Consider first $D, S, M, N, G$, and $L$. Algebraic tranformations yield that, for any $e, h \in \Gamma$ such that $s(e, h)=-1$,

$$
\begin{array}{rlrl}
D(e, h) & =Z(e, h) \cdot p(h) & D(h, e)=Z(h, e) \cdot p(e) \\
S(e, h) & =Z(e, h) \cdot[p(h) / p(\neg e)] & & S(h, e)=Z(h, e) \cdot[p(e) / p(\neg h)] \\
M(e, h) & =Z(e, h) \cdot p(e) & M(h, e)=Z(h, e) \cdot p(h) \\
N(e, h) & =Z(e, h) \cdot[p(e) / p(\neg h)] & N(h, e)=Z(h, e) \cdot[p(h) / p(\neg e)] \\
G(e, h) & =Z(e, h) \cdot[p(h)) / p(\neg h)] & G(h, e)=Z(e, h) \cdot[p(e)) / p(\neg e)] \\
L(e, h) & =\frac{Z(e, h)}{1-[p(h)-p(\neg h)] \cdot[1+Z(e, h)]} \\
L(e, h) & =\frac{Z(h, e)}{1-[p(e)-p(\neg e)] \cdot[1+Z(h, e)]} .
\end{array}
$$

By Lemma 2, for any $e, h \in \Gamma$ such that $s(e, h)=-1, Z(e, h)=Z(h$, $e$ ). Then, whenever $p(h) \neq p(e)$, each of the above measures (as well as any measure of confirmation ordinally equivalent to any of them) assigns a higher value to either $(e, h)$ or $(h, e)$, thus failing to mirror $I$ in case of disconfirmation, contrary to $\left(\mathrm{Ex}_{2}\right)$. 
Now consider the remaining measures $C$ and $R$. For any $e, h \in \Gamma$ such that $s(e, h)=1$,

$$
C(e, h)=p(h \& e)-p(h) \cdot p(e)=C(h, e)
$$

and

$$
R(e, h)=[p(h \mid e) / p(h)]-1=[p(e \mid h) / p(e)]-1=R(h, e) .
$$

Then, both $C$ and $R$ (as well as any measure of confirmation ordinally equivalent to any of them) mirror $I$ in case of confirmation, contrary to $\left(\mathrm{Ex}_{2}\right)$.

This completes the proof of part $\mathrm{b}$ and of the theorem.

\section{REFERENCES}

Carnap, R. ([1950] 1962), Logical Foundations of Probability. Chicago: University of Chicago Press.

Christensen, D. (1999), "Measuring Confirmation," Journal of Philosophy 96: 437-461.

Eells, E., and B. Fitelson (2002), "Symmetries and Asymmetries in Evidential Support," Philosophical Studies 107: 129-142.

Festa, R. (1999), "Bayesian Confirmation," in M. Galavotti and A. Pagnini (eds.), Experience, Reality, and Scientific Explanation. Dordrecht: Kluwer, 55-87.

Finch, H. A. (1960), "Confirming Power of Observations Metricized for Decisions among Hypotheses," Philosophy of Science 27: 293-307, 391-404.

Fitelson, B. (1999), "The Plurality of Bayesian Measures of Confirmation and the Problem of Measure Sensitivity," Philosophy of Science 66: S362-S378.

(2001), Studies in Bayesian Confirmation Theory. PhD dissertation, University of Wisconsin, Madison.

(2006), "Logical Foundations of Evidential Support," Philosophy of Science 73: 500512 .

(2007), "Likelihoodism, Bayesianism, and Relational Confirmation," Synthese 156: $473-489$.

Gaifman, H. (1979), "Subjective Probability, Natural Predicates and Hempel's Ravens," Erkenntnis 21: 105-147.

Girotto, V., and M. Gonzalez (2001), "Solving Probabilistic and Statistical Problems: A Matter of Question Form and Information Structure," Cognition 78: 247-276.

Good, I. J. (1950), Probability and the Weighing of Evidence. London: Griffin.

Hawthorne, J., and B. Fitelson (2004), "Re-solving Irrelevant Conjunction with Probabilistic Independence," Philosophy of Science 71: 505-514.

Horwich, P. (1982), Probability and Evidence. Cambridge: Cambridge University Press.

Joyce, J. (2004), "Bayes's Theorem," in E. N. Zalta (ed.), The Stanford Encyclopedia of Philosophy. Stanford, CA: Stanford University Press. http://plato.stanford.edu/archives/ sum2004/entries/bayes-theorem/.

Kahneman, D., P. Slovic, and A. Tversky, eds. (1982), Judgment under Uncertainty: Heuristics and Biases. New York: Cambridge University Press.

Kemeny, J., and P. Oppenheim (1952), "Degrees of Factual Support," Philosophy of Science 19: $307-324$

Keynes, J. (1921), A Treatise on Probability. London: Macmillan.

Kuipers, T. (2000), From Instrumentalism to Constructive Realism. Dordrecht: Reidel.

Marr, D. (1982), Vision: A Computational Approach. San Francisco: Freeman.

Mortimer, H. (1988), The Logic of Induction. Paramus, NJ: Prentice Hall.

Nozick, R. (1981), Philosophical Explanations. Oxford: Clarendon.

Osherson, D. N., E. E. Smith, O. Wilkie, A. Lopez, and E. Shafir (1990), "Category-Based Induction," Psychological Review 97: 185-200. 


\section{VINCENZO CRUPI, KATYA TENTORI, AND MICHEL GONZALEZ}

Rescher, N. (1958) "A Theory of Evidence," Philosophy of Science 25: 83-94.

Rips, L. J. (2001), "Two Kinds of Reasoning," Psychological Science 12: 129-134.

Sober, E. (1994), "No Model, No Inference: A Bayesian Primer on the Grue Problem," in D. Stalker (ed.), Grue! The New Riddle of Induction. Chicago: Open Court, 225-240.

Tentori, K., V. Crupi, N. Bonini, and D. Osherson (2007), "Comparison of Confirmation Measures," Cognition 103: 107-119. 